\title{
ULM'S THEOREM FOR TOTALLY PROJECTIVE GROUPS
}

\begin{abstract}
ELBERT A. WALKER ${ }^{1,2}$
ABSTRACT. This paper simplifies the proof of Hill's version of Ulm's theorem for totally projective groups by giving one which in essence is the same as the Mackey proof for the countable case.
\end{abstract}

1. Introduction. The classical theorem of UIm [11] characterizes countable reduced Abelian $p$-groups in terms of certain functions from ordinals to cardinals. Kolettis [8] extended Ulm's theorem to direct sums of such groups, and Parker and Walker [10] further extended it to totally projective groups of length less than $\Omega \omega$. Totally projective groups were defined by Nunke [9] via homological means. Hill gave an alternate definition and succeeded, in an admirable piece of work [5], [6] in extending Ulm's theorem to the class of all totally projective groups. Independently of the work of Parker and Walker, and of Hill, Crawley and Hales defined a $p$-group to be a $T$-group if it is generated by a set of elements subject only to relations of the form $p^{n} x=0$ and $p x=y$. They proved Ulm's theorem for reduced T-groups [1], [2], and using Hill's result, showed that the class of reduced $T$-groups coincides with the class of totally projective groups.

The simplest and most elegant proof of Ulm's theorem for countable reduced p-groups seems to be the one given by Kaplansky in [7]. He attributes it to Mackey. The proofs of the various generalizations above tend to be quite involved, particularly the extensions of Ulm's theorem to the class of totally projectives. The main purpose of this paper is to materially simplify the proof of Hill's version of Ulm's theorem for totally projective groups by giving one which in essence is the same as the Mackey proof for the countable case.

Throughout, $p$ is a fixed prime, and all groups are Abelian. We do not require the groups under consideration to be $p$-groups. For any group $G$ and ordinal $\alpha, p^{\alpha} G$ is defined inductively by $p^{\beta+1} G=p\left(p^{\beta} G\right)$, and $p^{\alpha} G=$ $\bigcap_{\beta<\alpha} p^{\beta} G$ for limit ordinals $\alpha$. For $g \in G$, the $p$-height of $g$, denoted

Received by the editors June 23, 1972.

AMS (MOS) subject classifications (1969). Primary 2030, 2027.

1 This research was supported by NSF grant GP-28379.

2 This paper is dedicated with appreciation to Professor Herbert O. Muecke. 
$\mathrm{ht}(g)$, is the smallest ordinal $\alpha$, if such exists, such that $g \notin p^{\alpha+1} G$. If $g \in p^{\alpha} G$ for all $\alpha$, then $h t(g)=\infty$, where $\infty$ is considered larger than any ordinal. The subgroup $G[p]=\{g \in G: p g=0\}$ is a vector space over the integers modulo $p$, and the dimension of $\left(p^{\alpha} G\right)[p] /\left(p^{\alpha+1} G\right)[p]$ is the ath Ulm invariant of $G$, denoted $u_{G}(\alpha)$. In the special case that $G$ is a direct sum $\sum_{i \in I} G_{i}$ of cyclic $p$-groups $G_{i}, u_{G}(n)$ is the number of summands $G_{i}$ of order $p^{n+1}$, and $u_{G}(\alpha)=0$ for infinite $\alpha$. A $p$-group $G$ is reduced if $p^{\alpha} G=0$ for some ordinal $\alpha$. The smallest such ordinal is the length of $G$. Ulm's theorem states that two countable reduced p-groups $G$ and $H$ are isomorphic if they have the same Ulm invariants, that is, if $u_{G}=u_{H}$. There exist reduced $p$-groups of every length, and countable ones of every length less than $\Omega$, the first uncountable ordinal. The reader may consult [3] or [7] for such standard facts about Abelian groups.

2. The proof of Ulm's theorem for totally projective groups. The concepts of nice subgroups (2.1) and relative Ulm invariants (2.5) introduced in this section, as well as the alternate definition (2.7) of totally projective groups, are due to Hill [5]. Hill's paper is essentially duplicated in [4]. We have modified Hill's definition of relative UIm invariant so that relative Ulm invariants are obviously invariant. Throughout, $p$ is a fixed prime, and all heights referred to are $p$-heights.

2.1. Definition [5]. A subgroup $A$ of $G$ is nice if every coset of $A$ has a representative of the same height, i.e., if for each $g \in G, \operatorname{ht}(g+A)=$ ht $(g+a)$ for some $a$ in $A$, where the two heights are computed in $G / A$ and in $G$, respectively.

If $A$ is nice in $G$, then certainly every coset of $A$ has a representative of maximum height. The converse is less obvious, but true.

2.2. Lemma. $A$ is nice in $G$ if and only if every coset of $A$ contains an element of maximum height.

Proof. Suppose that every coset of $A$ has an element of maximum height. If $A$ is not nice, let $g+A$ be a coset of minimum height such that $\mathrm{ht}(g+A)>\mathrm{ht}(g)$ and $g$ is of maximum height in $g+A$. Write $g+A=$ $p(x+A)$ with $\mathrm{ht}(x+A) \geqq \mathrm{ht}(\mathrm{g})$ and with $x$ of maximum height in $x+A$. Since $p x=g+a$, ht $(x)<\mathrm{ht}(g)$. Therefore $\mathrm{ht}(x)=\mathrm{ht}(x+A) \geqq \mathrm{ht}(g)$. This is a contradiction, and hence $A$ is nice in $G$. The converse is obvious, as noted.

If $g$ is of maximum height in $g+A$, then $g$ is said to be proper with respect to $A$.

2.3. COROllary. (a) Direct summands are nice. (b) For any ordina $\alpha, p^{\alpha} G$ is nice in $G$. (c) Finite subgroups are nice.

We need one additional fact about nice subgroups. 
2.4. Lemma. If $A$ is nice in $G$ and $B / A$ is nice in $G / A$, then $B$ is nice in G.

ProOF. ht $(g+B)=\mathrm{ht}(g+A+B / A)=\mathrm{ht}(g+b+A)=\mathrm{ht}(g+b+a)$ for suitable $a \in A, b \in B$.

Let $A$ be a subgroup of $G$, and let $\alpha$ be an ordinal. We denote $\left(p^{\alpha} G\right)[p] \cap$ $\left(A+p^{\alpha+1} G\right)$ by $A(\alpha)$. Note that $A(\alpha)=\{g \in G: p g=0$, ht $(g) \geqq \alpha$, and $\mathrm{ht}(g+a)>\alpha$ for some $a \in A\}$. The following definition is fundamental.

2.5. Definition. Let $A$ be a subgroup of $G$, and let $\alpha$ be an ordinal. The ath Ulm invariant of $G$ relative to $A$ is

$$
u_{G, A}(\alpha)=\operatorname{dim}\left(\left(p^{\alpha} G\right)[p] / A(\alpha)\right) .
$$

If $A=0$, then $u_{G, A}=u_{G}$, the ordinary Ulm function for $G$. This definition is equivalent to Hill's in [5].

Most of the technical details are taken care of by

2.6. Lemma. Suppose that $G / A$ and $H / B$ are reduced p-groups, $A$ and $B$ are nice subgroups of $G$ and $H$, respectively, and $\phi: A \rightarrow B$ is a height preserving isomorphism. Suppose that $u_{G . A}=u_{H . B}$, and let $f_{\alpha}:\left(p^{\alpha} G\right)[p] / A(\alpha) \rightarrow$ $\left(p^{\alpha} H\right)[p] / B(\alpha)$ be an isomorphism for each $\alpha$. If $x \in G$, then there are nice subgroups $A_{1}$ and $B_{1}$ of $G$ and $H$ respectively and $a$ height preserving isomorphism $\phi_{1}: A_{1} \rightarrow B_{1}$ extending $\phi$ such that

(a) $A_{1}=A+Z x$

(b) $A_{1} / A$ and $B_{1} / B$ are finite;

(c) for each $\alpha, f_{\alpha}$ induces an isomorphism $A_{1}(\alpha) / A(\alpha) \rightarrow B_{1}(\alpha) / B(\alpha)$; and

(d) $u_{G, A_{1}}=u_{H, B_{1}}$.

Proof. We may assume that $x \in A, p x \in A$, and that $x$ is proper with respect to $A$. If possible, $x$ is also chosen so that $h t(p x)>\operatorname{ht}(x)+1$. Let $\operatorname{ht}(x)=\beta$.

Case 1. ht $(p x)>\beta+1$. Let $p x=p y$ with ht $(y)>\beta$. Then $x-y$ has order $p$ and height $\beta$, and is proper with respect to $A$. Thus $0 \neq f_{\beta}(x-y+A(\beta))=$ $z+B(\beta)$, and so $z$ is of order $p$ and height $\beta$, and is proper with respect to $B$. Let $\phi(p x)=p w$ with ht $(w)>\beta$. Extend $\phi$ by sending $x$ to $w+z$. Let $A_{1}=$ $A+Z x$, and let $B_{1}=B+Z(w+z)$. The subgroups $A_{1}$ and $B_{1}$ are nice since $A_{1} / A$ and $B_{1} / B$ are finite and $A$ and $B$ are nice. If $\alpha \neq \beta$, then $A_{1}(\alpha)=A(\alpha)$ and $B_{1}(\alpha)=B(\alpha)$, and $f_{\alpha}$ certainly induces an isomorphism $A_{1}(\alpha) / A(\alpha) \rightarrow$ $B_{1}(\alpha) / B(\alpha)$. If $\alpha=\beta$, then $x-y \in A_{1}(\alpha)$, and $0 \neq f_{\alpha}(x-y+A(\alpha))=z+B(\alpha) \in$ $B_{1}(\alpha) / B(\alpha)$. Now $A_{1} / A$ has $p$ elements, and there is a natural epimorphism $A_{1} / A \rightarrow A_{1}(\alpha) / A(\alpha)$. Thus $\operatorname{dim}\left(A_{1}(\alpha) / A(\alpha)\right)=\operatorname{dim}\left(B_{1}(\alpha) / B(\alpha)\right)=1$, and $f_{\alpha}$ induces an isomorphism as required. This proves parts (a), (b), and (c). Part (d) follows from (c). 
Case 2. $\operatorname{ht}(p x)=\beta+1$. Let $\phi(p x)=p w$ with ht $(w) \geqq \beta$. Then ht $(w)=\beta$ since ht $(p w)=\beta+1$. If ht $(w+b) \geqq \beta+1$ with $b \in B$, then ht $(b)=\beta, b=\phi(a)$ with ht $(a)=\beta$, and ht $(x+a)=\beta$. Thus $x+a$ is proper with respect to $A$ and $\operatorname{ht}(p(x+a))>\beta+1$, and we are in Case 1. Hence $w$ is proper with respect to $B$. If $w \in B$, then $w=0$ and $h t(w)>h t(x)$. Extend $\phi$ by sending $x$ to $w$. Let $A_{1}=A+Z x$ and $B_{1}=B+Z w$. Again, if $\alpha>\beta$, then $x \in p^{\alpha+1} G$, and so $a+x+g+A(\alpha)=0$, and so $A_{1}(\alpha) / A(\alpha)=0=B_{1}(\alpha) / B(\alpha)$. If $\alpha=\beta$, then $\mathrm{ht}(a+x)=\beta$, and ht $(p(a+x))>\beta+1$ and we are in Case 1. Thus $A_{1}(\alpha) / A(\alpha)=0=B_{1}(\alpha) / B(\alpha)$, and our proof is complete.

2.7. Definition [5]. A reduced $p$-group $G$ is totally projective if it has a system $\mathscr{S}$ of nice subgroups such that

(a) $0 \in \mathscr{S}$;

(b) the subgroup generated by any subset of $\mathscr{S}$ is in $\mathscr{S}$;

(c) if $S \in \mathscr{S}$ and $A / S$ is countable, then there exists $B \in \mathscr{S}$ with $B \supseteq A$ and $B / A$ countable.

Countable reduced $p$-groups are totally projective. If $G$ is countable simply let $\mathscr{S}=\{0, G\}$. More generally, if $G=\sum_{i \in I} G_{i}$ with each $G_{i}$ countable, then $\mathscr{S}=\left\{\sum_{i \in J} G_{i}: J \subseteq I\right\}$ is a system of nice subgroups satisfying the conditions of 2.7. Thus direct sums of countable reduced $p$-groups are totally projective. Totally projective groups of arbitrary length are fairly easy to construct. Here is one way. For each ordinal $\alpha$, let $P_{\alpha}$ be the group with generators \{all formal products $\alpha \alpha_{1} \alpha_{2} \cdots \alpha_{n}: \alpha_{i}$ are ordinals, and $\left.\alpha>\alpha_{1}>\cdots>\alpha_{n}\right\}$ subject to the relations $p \alpha \alpha_{1} \cdots \alpha_{n} \alpha_{n+1}=\alpha \alpha_{1} \cdots \alpha_{n}$, and $p \alpha=0$. It is a fairly easy exercise to show that $p^{\alpha} P_{\alpha}=Z \alpha$ and is of order $p$, so that $P_{\alpha}$ has length $\alpha+1$. Noting that $P_{\alpha} / Z \alpha \cong \sum_{\beta<\alpha} P_{\beta}$, one may proceed inductively to show that $P_{\alpha}$ is totally projective. Indeed, direct sums of totally projective groups are totally projective ([4] or [5]), and if $\left\{S_{i} / Z \alpha\right\}_{i \in I}$ is a system of nice subgroups of $P_{\alpha} / Z \alpha$ satisfying the conditions of 2.7 , then 0 together with $\left\{S_{i}\right\}_{i \in I}$ is such a system for $P_{\alpha}$. Since $u_{P_{\alpha}}(\alpha)=1$, if $G$ is any totally projective group, then there is a collection of $P_{\alpha}$ 's whose direct sum $X$ has the same Ulm invariants as $X \oplus G$. Thus, Ulm's theorem for totally projective groups implies that every totally projective group is a direct summand of a direct sum of $P_{\alpha}$ 's. Conversely, direct summands of totally projective groups are totally projective: If $A \oplus B$ is totally projective and $\mathscr{S}$ is a system for $A \oplus B$, then $\mathscr{T}=\{X: X \subseteq A, X \oplus Y \in \mathscr{S}$ for some $Y \subseteq B\}$ is the desired system of nice subgroups for $A$ ([4] or [5]). In this connection, there is a serious gap in Griffith's proof $[4$, p. 74] that summands of totally projective groups are totally projective. Griffith uses a slightly different definition of totally projective. He replaces condition (b) in 2.7 by the weaker requirement that the system $\mathscr{S}$ be closed under unions of chains. With that definition, it is not clear that the system $\mathscr{T}$ above is closed under unions of chains. The two definitions in question are indeed 
equivalent. Our proof below of Ulm's theorem (2.9) is valid even if Griffith's weaker definition is used. The proof in [4] or [5] that direct sums of totally projective groups are totally projective works in both cases. Hence Griffith's (perhaps larger) class of totally projective groups also consists of summands of direct sums of $P_{\alpha}$ 's, whence the two definitions are equivalent. It would be curious to see a simple direct proof of this fact. Probably none exists.

We come now to the main theorem. The theorem itself is the same as Hill's in [5], except for the possibly significant fact that $\mathrm{G}$ and $H$ below are not required to be $p$-groups.

2.8. THEOREM. Let $G \mid A$ and $H \mid B$ be totally projective with $A$ and $B$ nice subgroups with $u_{G, A}=u_{H, B}$. Then any height preserving isomorphism $\phi: A \rightarrow B$ extends to an isomorphism $G \rightarrow H$.

Proof. Let $f_{\alpha}: p^{\alpha} G[p] / A(\alpha) \rightarrow p^{\alpha} H[p] / B(\alpha)$ be an isomorphism for each ordinal $\alpha$. Let $\mathscr{C}$ and $\mathscr{D}$ be systems of nice subgroups of $G / A$ and $H / B$, respectively, satisfying the conditions of 2.7 . Let $\mathscr{F}$ be the family of all height preserving isomorphisms $C \rightarrow D$ extending $\phi$ such that

(a) $C / A \in \mathscr{C}, D / B \in \mathscr{D}$, and

(b) for each $\alpha, f_{\alpha}$ induces an isomorphism $C(\alpha) / A(\alpha) \rightarrow D(\alpha) / B(\alpha)$.

Zorn's lemma applies in the obvious way, so there is a maximal such $\phi_{0}: C \rightarrow D$. Condition (b) yields $u_{G, C}=u_{H, D} . C$ and $D$ are nice by 2.4 , and for each $\alpha, f_{\alpha}$ induces an isomorphism $\left(p^{\alpha} G\right)[p] / C(\alpha) \rightarrow\left(p^{\alpha} H\right)[p] / D(\alpha)$. Suppose $G \neq C$, and let $g \in G \backslash C$. By 2.6 there are nice subgroups $A_{1}=C+$ $Z g$ and $B_{1}$, and a height preserving isomorphism $A_{1} \rightarrow B_{1}$ extending $\phi_{0}$ such that $f_{\alpha}$ induces an isomorphism $A_{1}(\alpha) / C(\alpha) \rightarrow B_{1}(\alpha) / D(\alpha)$, and hence an isomorphism $A_{1}(\alpha) / A(\alpha) \rightarrow B_{1}(\alpha) / B(\alpha)$. Now $A_{1} \subset C_{1}$ with $C_{1} / A \in \mathscr{C}$ and with $C_{1}=A_{1}+\sum_{i=1}^{\infty} Z x_{1 i}$. There is a height preserving isomorphism $A_{1}+$ $Z x_{11} \rightarrow B_{2}$ satisfying condition (b), and extending the isomorphism $A_{1} \rightarrow B_{1}$. Now $B_{2} \subset D_{2}=B_{2}+\sum_{i=1}^{\infty} Z y_{2 i}$ with $D_{2} / B \in \mathscr{D}$, and there is a height preserving isomorphism $A_{2} \rightarrow B_{2}+Z y_{21}$ that extends our previous one and which satisfies condition (b). Now $A_{2} \subset C_{2}=A_{2}+\sum_{i=1}^{\infty} Z x_{2 i}$ with $C_{2} / A \in \mathscr{C}$. There is a height preserving isomorphism $A_{2}+Z x_{12}+Z x_{21} \rightarrow B_{3}$ extending our previous one and satisfying condition (b). Now $B_{3} \subset D_{3}=B_{3}+$ $\sum_{i=1}^{\infty} Z y_{3 i}$ with $D_{3} / B \in \mathscr{D}$. There is a height preserving isomorphism $A_{3} \rightarrow$ $B_{3}+Z y_{22}+Z y_{31}$ extending our previous one and satisfying condition (b). Continuing in this manner we get a height preserving isomorphism $\bigcup C_{i}=\bigcup A_{i} \rightarrow \bigcup D_{i}$ extending $\phi_{0}$ and satisfying condition (b). But $\left(\bigcup C_{i}\right) / A \in \mathscr{C}$ and $\left(\bigcup D_{i}\right) / B \in \mathscr{D}$. We conclude that $C=G$. Similarly $B=H$, and the proof is complete.

2.9. COROLLARY. Two totally projective groups are isomorphic if they have the same Ulm invariants. 
2.10. Corollary. Suppose that $G$ and $H$ are torsion free groups and $A$ and $B$ are nice subgroups of $G$ and $H$, respectively, such that $G / A$ and $H / B$ are totally projective. Then any height preserving isomorphism from $A$ to $B$ extends to an isomorphism from $G$ to $H$.

\section{REFERENCES}

1. P. Crawley and A. W. Hales, The structure of torsion abelian groups given by presentations, Bull. Amer. Math. Soc. 74 (1968), 954-956. MR 38 \#1163.

2. - The structure of abelian p-groups given by certain presentations, J. Algebra 12 (1969), 10-23. MR 39 \#307.

3. L. Fuchs, Abelian groups, Internat. Series of Monographs on Pure and Appl. Math., Pergamon Press, New York, 1960. MR 22 \#2644.

4. P. Griffith, Infinite abelian group theory, Univ. of Chicago Press, Chicago, Ill., 1970.

5. P. Hill, On the classification of abelian groups (to appear).

6. - Ulm's theorem for totally projective groups, Notices Amer. Math. Soc. 14 (1967), 940. Abstract \#652-15.

7. I. Kaplansky, Infinite abelian groups, Univ. of Michigan Press, Ann Arbor, Mich., 1969. MR 38 \#2208.

8. G. Kolettis, Jr., Direct sums of countable groups, Duke Math. J. 27 (1960), 111125. MR 22 \#1616.

9. R. Nunke, Homology and direct sums of countable abelian groups, Math. Z. 101 (1967), 182-212. MR 36 \#1538.

10. L. D. Parker and E. A. Walker, An extension of the Ulm-Kolettis theorems, Studies on Abelian Groups (Sympos., Montpellier, 1967), 309-325. MR 42 \#367.

11. H. Ulm, Zur Theorie der Abzahlbar-unendlichen Abelschen Gruppen, Math. Ann. 107 (1933), 774-803.

Department of Mathematics, New Mexico State University, Las Cruces, New MeXICO 88003 